\title{
Objective Quantification of Image Quality and Optical Scatter Before and After Nd:YAG Capsulotomy Using a Double-Pass Technique
}

This article was published in the following Dove Press journal: Clinical Ophthalmology

\author{
Abu-Bakar Zafar' \\ Richard C Chu ${ }^{2}$ \\ Miranda N Bishara ${ }^{3}$ \\ Mujtaba A Qazi (iD ${ }^{4,5}$ \\ Ashraf M Mahmoud ${ }^{6}$ \\ Jay $S$ Pepose $\mathbb{I D}^{4,5}$
}

'Department of Ophthalmology, Carle Foundation Hospital, Champaign, IL, USA; ${ }^{2}$ Chu Eye Institute, Fort Worth, TX, USA; ${ }^{3}$ Cokingtin Eye Center, Overland Park, KS, USA; ${ }^{4}$ Pepose Vision Institute, St. Louis, MO, USA; ${ }^{5}$ Department of Ophthalmology and Visual Sciences, Washington University School of Medicine, St. Louis, MO, USA; ${ }^{6}$ Department of Ophthalmology, Ohio State University, Columbus, OH, USA
Correspondence: Jay S Pepose

Pepose Vision Institute, 1815 Clarkson Road, Chesterfield, MO 63107, USA

Email jpepose@peposevision.com
Purpose: The purpose of this study is to evaluate and compare the correlation between changes in vision and HD Analyzer dual-pass metrics versus changes in vision and conventional subjective slit lamp gradings in pseudophakic patients with posterior capsular opacity undergoing neodymium:yttrium-aluminum-garnet (Nd:YAG) capsulotomy.

Patients and Methods: High contrast (HC) and low contrast (LC) best spectacle-corrected distance visual acuity (BCVA) and HD Analyzer evaluation were prospectively performed on patients with mild-to-moderate posterior capsular opacification (PCO) and monofocal and accommodating intraocular lens implants. Differences between pre- and post-operative measurements were calculated, along with the correlation of HD Analyzer metrics and slit lamp grading to changes in visual acuity.

Results: Following Nd:YAG capsulotomy $(n=29)$, there was statistically significant improvement in HC-BCVA and LC-BCVA, decrease in optical scatter, and corresponding improvement in Strehl ratio and HD Analyzer values at all contrast levels tested $(\mathrm{p} \leq 0.05)$. Pearson test showed a high correlation between the improvement in HC-BCVA ( $\mathrm{r}$ coefficient $=0.78)$ and LC-BCVA ( $\mathrm{r}$ coefficient $=0.71)$ to the improvement in Objective Scatter Index (OSI). There was a higher correlation of change in HC-BCVA to pre-op OSI $\left(\mathrm{r}^{2}=0.61\right)$ than to the subjective PCO grading score $\left(r^{2}=0.19\right)$. There was also a higher correlation of change in LC-BCVA to pre-op OSI $\left(r^{2}=0.49\right)$ than to subjective grading $\left(r^{2}=0.16\right)$.

Conclusion: The HD Analyzer provides objective measurements of forward light scatter (ie, light directed towards the retina) that can assist with both PCO grading and prediction of improvement of visual quality after YAG laser capsulotomy with higher accuracy than conventional slit lamp assessment based upon backscatter (ie, light traveling to the observer) in patients tested with monofocal and accommodating intraocular lens implants.

Keywords: HD Analyzer, posterior capsular opacity, YAG capsulotomy, objective scatter index

\section{Introduction}

Given the increasing prevalence of cataract surgery in countries with an aging population, the consequent prevalence of posterior capsular opacification (PCO) is also anticipated to increase. ${ }^{1}$ PCO development is due to the proliferation of residual lens epithelial cells along the lens capsule behind the intraocular lens (IOL), affecting decreased visual acuity, glare disability, and lowered contrast sensitivity. Fibrotic PCO can result from transdifferentiation of residual lens epithelial cells into myofibroblasts, causing fibrosis and contraction of the capsular bag, as well as IOL decentration or tilt. ${ }^{2}$ Although refinements in surgical techniques and 
square edge IOL designs have played a role in the reduction of PCO formation, it remains one of the most common sequelae following cataract surgery. PCO can be treated via neodymium:yttrium-aluminum-garnet $(\mathrm{Nd}$ : YAG) laser capsulotomy or, less commonly, via surgical capsulotomy. Laser capsulotomy is not without complications. Induction of cystoid macular edema, increased intraocular pressure, floaters, iritis, intraocular lens tilt, pitting or displacement, and increased incidence of retinal hemorrhage, retinal tear, and detachment are all potential risks of this procedure. ${ }^{3,4}$

The decision to perform posterior capsulotomy is frequently based on subjective symptoms and/or decreased visual acuity. Brightness acuity testing (BAT), neutral density filtered acuity, contrast sensitivity, and glare assessment are ancillary tests that can be used to evaluate the impact of PCO on visual symptoms. Subjective evaluation and grading of PCO allows for wide inter- and intra-observer variation. The use of only slitlamp retroillumination has been shown to underestimate the presence and severity of PCO. ${ }^{5}$ Thus, PCO morphology and impact on visual quality may not be adequately evaluated with slit-lamp assessment. Slit-lamp examination and imaging captures the backscatter of light off the anterior PCO surface but does not directly assess the forward scatter that results as light passes through the PCO towards the retina, which is what the patient experiences. Computer-based software systems (ie, Automated Quantification of AfterCataract (AQUA), Evaluation of Posterior Capsular Opacification (EPCO), PearlTracer, and Posterior Capsular Opacification (POCO)), ${ }^{6-10}$ provide reproducible and objective measures of the PCO using digital images without directly assessing the impact of the PCO on the visual system. ${ }^{11}$ Furthermore, these systems are not readily available in the clinical setting and are based upon backscatter. Rotating Scheimpflug imaging has been shown to give reproducible measurements quantifying $\mathrm{PCO},{ }^{12,13}$ but the scatter light density measurements with this system are substantially influenced by IOL material, which may limit the direct comparison of PCO in IOLs of different materials. $^{14}$

The HD Analyzer (Visiometrics by Keeler; Malvern, PA) ${ }^{15-18}$ (Figure 1) measures the retinal point spread function via a double-pass method. A 780-nm collimated diode laser passes through a 2-mm aperture onto the retina. The reflected light then travels through a 4-mm exit aperture prior to reaching a charge-coupled device (CCD) camera. The measured retinal point spread function (Figure 2) is used to

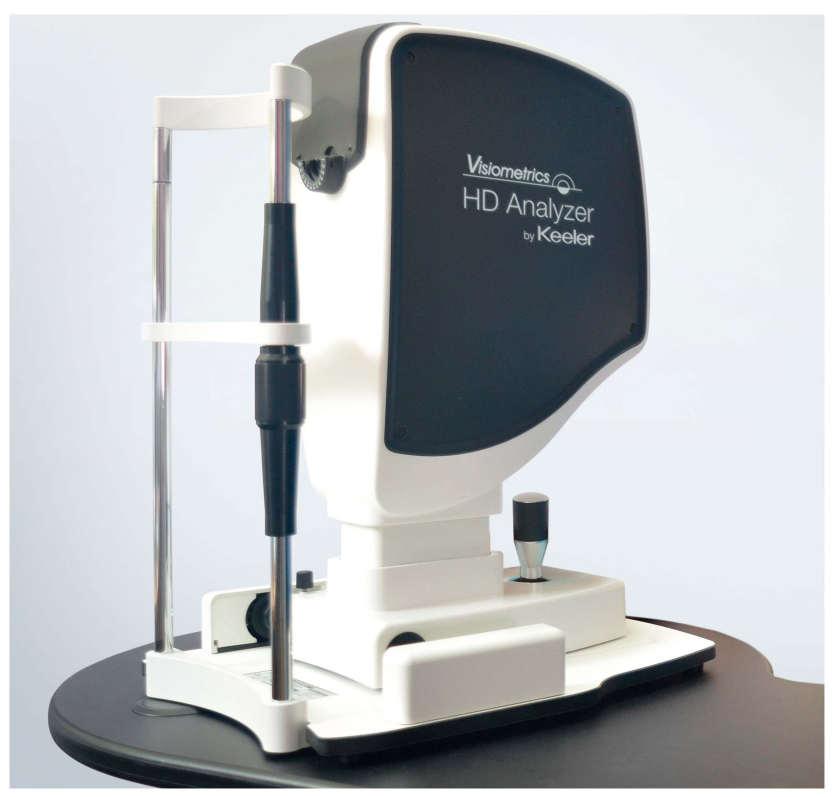

Figure I The Visiometrics HD Analyzer (image courtesy of Keeler; Malvern, PA) measures the retinal point spread function via a double-pass method, providing an objective measure of forward light scatter and other metrics of visual quality.

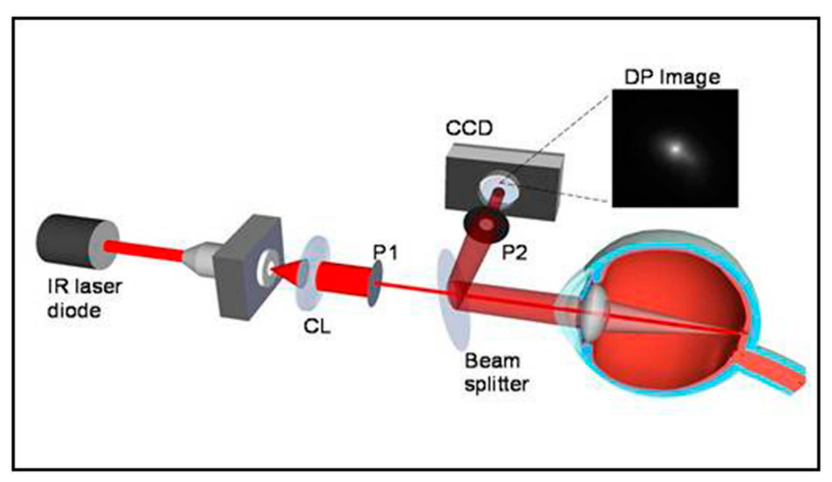

Figure 2 The HD Analyzer uses an infrared laser and a double-pass image through asymmetric entrance and exit apertures captured with a charge-coupled device camera, allowing inference of the complete optical transfer function of the eye as well as the shape of the retinal point spread function.

calculate the Strehl ratio, Modulation Transfer Function (MTF), MTF cutoff value, Objective Scatter Index (OSI), and HD Analyzer contrast sensitivity values (OV).

The Modulation Transfer Function's value indicates how closely an object is captured in the retinal image as a function of spatial frequency. The MTF is a ratio of image to object contrast and can be degraded by the effects of scatter, diffraction, and lower- and higherorder aberrations. The MTF cutoff value is the maximal spatial frequency that can be resolved by an optical system. The Strehl ratio, ranging from zero to one, compares the calculated MTF to that of a diffraction-limited system, 
with a value of one corresponding to an unaberrated optical system. The HD Analyzer values are derived from the MTF and describe the patient's visual quality with contrasts of $100 \%, 20 \%$, and $9 \%$, as well as an averaged value (Mean OV). The $\mathrm{OSI}^{18}$ is measured from the signal intensity of the peripheral region of the retinal PSF image relative to the signal intensity of the central region. Thus, the higher the OSI number, the greater the amounts of intraocular and/or ocular surface scatter.

The light scatter measured by the HD Analyzer can occur anywhere along the optical pathway. Even in a young healthy patient, light scatter, albeit minimal, occurs at the tear film, the cornea, the iris or pupil margin, the lens, the vitreous media, and at the retina. Dry eye states, ${ }^{19-21}$ corneal $^{22,23}$ disease or haze, cataract, ${ }^{24-27}$ and vitreous turbidity or opacities (eg, asteroid hyalosis) can all cause increased light scatter. Thus, the HD Analyzer has been used to assess retinal image quality in patients with cataracts, pseudophakia, and phakic IOLs. Multiple studies ${ }^{28-30}$ have shown good reproducibility for measurements using the HD Analyzer system. This study applies the HD Analyzer metrics to the evaluation of PCO and the improvements in visual function after laser capsulotomy.

\section{Patients and Methods}

Patients scheduled for Nd:YAG laser capsulotomy by one surgeon (MQ) were consecutively enrolled in this prospective study after appropriate informed consent was obtained for participation in the study. All investigations were conducted in a single site in the US in accordance with the Health Insurance Portability and Accountability Act regulations and the tenets of the Declaration of Helsinki and were approved by the Southwest Independent Institutional Review Board (Fort Worth, Texas). All subjects provided written informed consent.

Patients with any history of ocular pathology, including corneal disease and opacities, moderate to severe dry eyes, macular or optic nerve disease, and/or previous ocular surgery, other than uncomplicated cataract surgery with in-the-bag IOL implantation, were excluded. Patients that had pupil diameter less than $4 \mathrm{~mm}$ in mesopic conditions were also excluded, as a minimum of $4 \mathrm{~mm}$ pupil was required for valid HD Analyzer measurement. Patients with multifocal, extended depth of focus, or toric IOLs were also not included.

High contrast distance uncorrected visual acuity (HC-UCVA) and best-spectacle corrected distance visual acuity (HC-BCVA) on an Early Treatment of Diabetic
Retinopathy Study (ETDRS) chart, low contrast best-spectacle corrected visual acuity (LC-BCVA, Optec II, Stereo Optical Co, Inc., Chicago, Illinois, USA), and HD Analyzer evaluation were performed on patients with PCO preoperatively and 2 to 4 weeks postoperatively. Additionally, the average of subjective grading of PCO by two observers (MQ, MB) was calculated using the following scale: ${ }^{31}$

- $0=$ no opacity;

- $1=$ wrinkling or opacity of the capsule within a central 4 $\mathrm{mm}$ diameter, with no degradation of the retinal view;

- $2=$ central/paracentral opacity within central $4 \mathrm{~mm}$ diameter sufficient to degrade details of the macula, but still permitting optic nerve cup/disc ratio assessment;

- $3=$ central/paracentral opacity sufficient to make cup/ disc ratio assessment difficult, or moderate reduction of red reflex;

- $4=$ central/paracentral opacity sufficient to make visualization of fundus details difficult or impossible, or severe reduction of red reflex.

HD Analyzer data were captured with a 4-mm exit aperture in undilated eyes. When necessary, patients were darkadapted to achieve a minimum of $4 \mathrm{~mm}$ pupillary aperture. The spherical refractive error was measured and corrected by the motorized optometer within the HD Analyzer. External cylindrical trial lenses were used to correct astigmatism greater than $0.5 \mathrm{D}$. Uniform mesopic room illumination was employed for all testing. A single artificial teardrop (Refresh Plus, Allergan, Inc., Irvine, California, USA) was instilled into the eye 10 minutes prior to measurement of the HD Analyzer visual parameters to obviate scatter contributed by dry eye, per standard protocol for HD Analyzer image capture. OSI, Retinal Image curve profile width at 50\% and $10 \%$ height, Strehl ratio, and HD Analyzer contrast values $(\mathrm{OV})$ at $100 \%, 20 \%$, and $9 \%$ contrast were recorded.

Differences between pre- and post-operative measurements were calculated, along with Pearson correlation of HD Analyzer metrics to improvement in visual acuity. Paired Student's $t$-tests, with $\mathrm{p}$ value $\leq 0.05$ being statistically significant, were used for statistical comparisons of pre- to post-operative measurements.

\section{Results}

\section{Demographics}

Twenty-nine eyes of 25 ( 12 male and 13 female) patients age 46 to 81 (mean $64.5 \pm 8.6 \mathrm{yrs}$ ) were included. The average 
time from cataract surgery to YAG was 563 days (107-1557 days). Nineteen of the 29 had Crystalens-AT accommodating IOL (5.0 mm optic, Bausch \& Lomb Surgical, Rancho Cucamonga, CA, USA) and 10 had monofocal aspheric (6.0 mm optic) IOLs (SoftPort, Bausch \& Lomb Surgical, Rancho Cucamonga, CA, USA). Some Crystalens patients had mild central PCO with wrinkling or signs of early capsular contraction and underwent $\mathrm{Nd}$ :YAG laser capsulotomy in an effort to avoid the occurrence of a z-syndrome (ie, asymmetric vaulting of the haptics and optic of a plate haptic IOL). ${ }^{32}$ There were 5 eyes with a subjective PCO grade of 1 , 14 with grade 2, 10 with grade 3, and none with grade 4 . There was 93\% agreement of the subjective PCO grading between the two observers, with two eyes having a difference of 1 scale score (in which case, the lower score was utilized).

\section{Visual Acuity}

There was an improvement in mean values for all high and low contrast visual parameters following YAG capsulotomy, which were statistically significant (Table 1). The mean improvement in HC-UCVA and HC-BCVA was $0.07 \pm 0.17 \log$ MAR $(p=0.049)$ and $0.06 \pm 0.11 \log$ MAR $(p=0.021)$, respectively. A clinically greater mean improvement of $0.12 \pm 0.18 \log \operatorname{MAR}(\mathrm{p}=0.001)$ was detected by measuring LC-BCVA. No eyes had worsening of HC-BCVA following YAG capsulotomy.

Eyes with a subjective PCO grade of $2(n=14)$ had on average an approximately one line improvement in LC-BCVA (mean $\Delta=-0.08 \pm 0.13 \log$ MAR, $p=0.043$ ) and a smaller but statistically significant improvement in HC-BCVA (mean $\Delta=-0.03 \pm 0.05 \log$ MAR, $\mathrm{p}=0.04$ ) following YAG capsulotomy. Those with a subjective PCO grade of $3(n=10)$ had on average over one line improvement in HC-BCVA (mean $\Delta=-0.13 \pm 0.16 \log$ MAR, $\mathrm{p}=0.1$ ) and greater than 2 line improvement in LC-BCVA (mean $\Delta=$ $-0.22 \pm 0.24 \log$ MAR, $\mathrm{p}=0.016$ ).

Table I Visual Acuity Parameters with YAG Capsulotomy ( $I$ Line Improvement in Acuity $=-0.10 \log$ MAR)

\begin{tabular}{|l|l|l|l|l|}
\hline $\begin{array}{l}\text { Visual } \\
\text { Acuity } \\
\text { (log MAR) }\end{array}$ & Pre-YAG & Post-YAG & $\begin{array}{l}\text { Change (Post } \\
- \text { Pre) }\end{array}$ & $\begin{array}{l}\text { P } \\
\text { value }\end{array}$ \\
\hline HC-UCVA & $+0.22 \pm 0.23$ & $+0.16 \pm 0.20$ & $-0.07 \pm 0.17$ & 0.049 \\
HC-BCVA & $+0.01 \pm 0.15$ & $-0.04 \pm 0.07$ & $-0.06 \pm 0.11$ & 0.021 \\
LC-BCVA & $+0.35 \pm 0.17$ & $+0.23 \pm 0.11$ & $-0.12 \pm 0.18$ & 0.001 \\
\hline
\end{tabular}

Abbreviations: HC, high contrast; LC, low contrast; UCVA, uncorrected visual acuity; BCVA, best spectacle-corrected visual acuity.
As mentioned previously, a number of YAG capsulotomies $(n=13)$ were performed in Crystalens patients with minimal posterior capsular fibrosis (pre-YAG OSI $\leq 1$ ), but signs of early capsular contraction, where the capsulotomy was performed predominantly to prevent a z-syndrome. For eyes with a pre-YAG OSI greater than 1.0 (mean $3.48 \pm 3.05, \mathrm{n}=16$ ), HC-BCVA improved by an average of approximately one line (mean $\Delta=-0.09 \pm 0.14 \mathrm{log}$ MAR, $p=0.055)$ and LC-BCVA improved by an average of two lines (mean $\Delta=-0.20 \pm 0.20 \log$ MAR, $\mathrm{p}=0.001$ ) following surgery. If the pre-YAG OSI was 2 or greater (mean $6.03 \pm 3.10, n=7$ ), there was on average an approximately two line improvement of HC-BCVA (mean $\Delta=$ $-0.19 \pm 0.17 \log$ MAR, $\mathrm{p}=0.026)$ and approximately a three line improvement of LC-BCVA (mean $\Delta=-0.29 \pm$ $0.25 \log$ MAR, $\mathrm{p}=0.025$ ).

\section{HD Analyzer Metrics}

Improvement in the OSI, MTF cutoff, Strehl ratio, and mean contrast HD Analyzer parameters after intervention with YAG capsulotomy were all statistically significant (Table 2). On average, OSI was 1.35 lower $(p=0.007)$ after YAG capsulotomy. Eyes with a PCO grade of 3 had a greater improvement in OSI (mean $\Delta$ OSI $=-2.95$, $\mathrm{n}=10, \mathrm{p}=0.023$ ) than those with a grade 2 (mean $\Delta$ OSI $=-1.65, n=14, p=0.006)$. All OV metrics improved after surgical intervention, with improvement of the Mean OV by $0.36 \pm 0.38(\mathrm{p}<0.001)$. The highest correlation of change in HC-BCVA to any HD Analyzer metric was to the change of OSI (Pearson $=+0.78$ ), which was higher than the correlation of change in OSI to PCO grade $($ Pearson $=-0.44)$.

\section{Correlations}

While the mean OSI scores increased with increasing subjective grading of PCO, there was some overlap in OSI values between Grades 2 and $3(\mathrm{p}=0.07)$ (Figure 3). OSI scores were significantly lower for Grade 1 (mean 0.6 ) than both Grade 2 (mean 1.6, p=0.04) and 3 (mean 3.9, p=0.02).

There was a very high correlation between pre-OSI and change in OSI (linear regression $r^{2}=0.98$ ). Both pre-YAG OSI $\left(\mathrm{r}^{2}=0.61\right)$ and the change in OSI $\left(\mathrm{r}^{2}=0.60\right)$ had a higher correlation to change in HC-BCVA (Table 3 ) than subjective grade $\left(\mathrm{r}^{2}=0.19\right)$ and other HD-Analyzer parameters (Table 2, Pearson). The linear regression equation [preYAG OSI $=-18.46(\triangle \mathrm{HC}-\mathrm{BCVA} \log \mathrm{MAR})+1.08$ ] comparing pre-YAG OSI to $\triangle \mathrm{HC}$-BCVA predicts at least one line improvement $(-0.1 \log \mathrm{MAR})$ in $\mathrm{HC}-\mathrm{BCVA}$ if the pre-YAG 
Table 2 HD Analyzer Parameters Pre- and Post-YAG Capsulotomy

\begin{tabular}{|l|l|l|l|l|l|l|l|}
\hline $\begin{array}{l}\text { HD Analyzer } \\
\text { Parameter }\end{array}$ & Pre-YAG & Post-YAG & $\begin{array}{l}\text { Change (Post } \\
- \text { Pre) }\end{array}$ & $\begin{array}{l}\mathbf{P} \\
\text { value }\end{array}$ & $\begin{array}{l}\text { Pearson of } \\
\Delta \text { parameter to } \\
\Delta \text { HC-BCVA }\end{array}$ & $\begin{array}{l}\text { Pearson of } \\
\Delta \text { parameter to } \\
\Delta \text { LC-BCVA }\end{array}$ & $\begin{array}{l}\text { Pearson of } \\
\Delta \text { parameter to } \\
\text { PCO Grade }\end{array}$ \\
\hline OSI & $2.23 \pm 2.64$ & $0.88 \pm 0.40$ & $-1.35 \pm 2.49$ & 0.007 & +0.78 & +0.71 & -0.44 \\
MTF Cutoff & $26.44 \pm 11.92$ & $37.25 \pm 7.68$ & $+10.81 \pm 13.55$ & $<0.001$ & -0.34 & -0.54 & +0.15 \\
Strehl & $0.14 \pm 0.04$ & $0.18 \pm 0.04$ & $+0.05 \pm 0.05$ & $<0.001$ & -0.29 & -0.42 & +0.18 \\
Width @ 50\% & $6.09 \pm 4.62$ & $3.37 \pm 0.85$ & $-2.72 \pm 4.67$ & 0.004 & +0.64 & +0.54 & -0.25 \\
Width @ 10\% & $17.53 \pm 10.96$ & $12.33 \pm 3.82$ & $-5.20 \pm 11.02$ & 0.024 & -0.08 & -0.01 & -0.10 \\
\hline
\end{tabular}

Notes: Width, point spread function profile width at 10\% (arc min) and 50\% (arc min) of the maximum height; $\Delta$, change (post-pre).

Abbreviations: PCO, posterior capsular opacity; OSI, Objective Scatter Index; MTF, Modulation TransferFunction.

OSI is 2.93 or greater. When considering $\triangle \mathrm{LC}-\mathrm{BCVA}$, the regression equation [pre-YAG OSI=-10.36 ( $\triangle \mathrm{LC}-\mathrm{BCVA}$ $\log \mathrm{MAR})+0.98]$ predicts at least one line improvement in LC-BCVA if the pre-YAG OSI is 2.02 or greater.

\section{Discussion}

In this study, we demonstrated the utility of using the HD Analyzer to objectively quantify optical scatter and other metrics of visual quality before and after Nd:YAG capsulotomy. There was improvement in both high and low contrast BCVA with the latter able to detect a more clinically significant change, a decrease in optical scatter (mean OSI pre-YAG 2.23 to post-YAG 0.88 ; $\mathrm{p}=0.007$ ), and a corresponding improvement in Strehl ratio and HD Analyzer values at all contrast levels tested. Of import, the Pearson test showed a high correlation between the improvement in HC-BCVA ( $\mathrm{r}$ coefficient=0.78) and LC-BCVA ( $r$ coefficient=0.71) to the improvement in OSI. In distinction to subjective slit lamp grading of $\mathrm{PCO}$, which is generally considered the current clinical standard of care, there was a higher correlation of objective measures, namely the pre-op OSI $\left(\mathrm{r}^{2}=0.61\right)$ and change in OSI $\left(r^{2}=0.60\right)$ to change in HC-BCVA, while the correlation of the subjective grading score to change in HC-BCVA was substantially lower $\left(\mathrm{r}^{2}=0.19\right)$.

Recently, Zhang and $\mathrm{Wang}^{33}$ also showed a strong correlation between the OSI metric and HC-BCVA. They noted that several patients had a subjective improvement following Nd:YAG capsulotomy based upon responses to a questionnaire grading visual disturbance, along with a decrease in OSI, but no improvement in BCVA. As we studied the effect of YAG laser capsulotomy on a number of patients with mild capsular fibrosis, where the procedure was performed in an effort to prophylactically prevent a $z$-syndrome in an accommodating IOL, ${ }^{32}$ we similarly saw improvements in LC-BCVA in some eyes, but not always HC-BCVA. These findings are supported by the work of Yotsukura and colleagues, ${ }^{34}$ who noted improvement in the quality of vision as assessed by LCVA, functional visual acuity, and wavefront aberrations

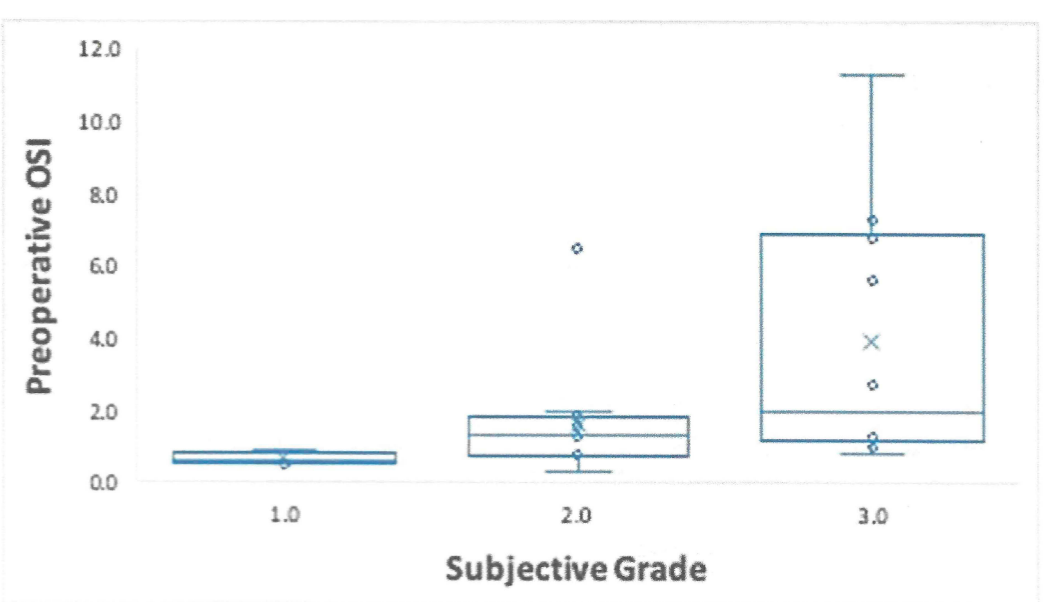

Figure 3 Preoperative OSI for each subjective PCO grading. Preoperative OSI values were significantly lower for Grade I than for both Grade 2 ( $=0.0392$ ) and Grade 3 $(p=0.0184)$ and approached statistical significance when comparing values between Group 2 and $3(p=0.0782)$. 
Table 3 Correlations $\left(R^{2}\right)$ Between Change in High and Low Contrast Visual Acuity and Objective and Subjective Metrics

\begin{tabular}{|l|l|l|}
\hline & $\begin{array}{l}\mathbf{R}^{2} \text { to } \\
\Delta \mathbf{H C}-B C V A\end{array}$ & $\begin{array}{l}\mathbf{R}^{2} \text { to } \\
\Delta \mathbf{L C}-B C V A\end{array}$ \\
\hline Pre-YAG OSI & 0.61 & 0.49 \\
$\Delta$ OSI & 0.60 & 0.49 \\
Grade & 0.19 & 0.16 \\
\hline
\end{tabular}

Note: $\Delta$, change (post-pre).

Abbreviations: HC-BCVA, high contrast best-corrected visual acuity; LC-BCVA, low contrast best-corrected visual acuity; OSI, Objective Scatter Index.

in patients following Nd:YAG capsulotomy who did not demonstrate an improvement in HC-BCVA.

The poor correlation that we found between the OSI (and other objective HD Analyzer quality of vision metrics) and the subjective slit lamp gradings of PCO is consistent with the findings of Hirnschall et al. ${ }^{6}$ In studying subjects with PCO, they used 2 measurements of forward light scatter and 2 of backscattered light and found that the backward and forward light scattering measurements did not correlate well. One possible explanation for this discrepancy is that backward light scatter as seen at the slit lamp depends on larger angles between the direction of illumination and observation and also, at very large angles, involves reflectance, whereas forward scatter (as seen by the patient) is dominated by smaller angles $\left(<28\right.$ degrees) ${ }^{35}$

The subjectivity of PCO grading at the slit lamp may lead to inter- and intra-observer discrepancies. Photographic and automated image analysis techniques permit more objective assessment of the location, dimensions, and severity of PCO, as well as document changes over time, by capturing light reflected off of the anterior PCO surface or via retroillumination techniques. Some have fused images to obviate unwanted reflections or Purkinje images that hamper the analysis. ${ }^{36}$ However, these systems rely on imaging derived from backscattered light and do not directly measure the forward scatter of light focused onto the fovea, which is what the patient sees. $^{7-9}$ This may partly explain why analysis of retroillumination images alone may cause underestimation of PCO. ${ }^{5}$ Other subjective psychovisual testing systems such as the C-Quant straylight meter (Oculus, Wetzlar, Germany) provide qualitative data, are time consuming to perform relative to other testing methods, and cannot be used in patients with limited visual acuity. ${ }^{6}$ Also, studies indicate limited correlation between Straylight values and visual improvement after Nd:YAG capsulotomy. ${ }^{37}$
More recent studies by these investigators suggest that qualitative straylight thresholds may better predict clinically significant improvement in visual acuity on patients with low PCO severity. ${ }^{38}$

$\mathrm{Lu}$ and colleagues ${ }^{39}$ used the RTVue-100 OCT (Opotovue, Inc., Fremont, CA) to evaluate PCO morphology and severity. They showed that PCO morphology (pear type versus fibrosis) affected OSI scores and found that changes in OSI following YAG capsulotomy were correlated to the PCO area in the central $3 \mathrm{~mm} \mathrm{IOL}$ optic region $(r=0.43, p=0.02)$. For all eyes, regardless of morphology, there was a positive correlation (0.67, $\mathrm{p}<0.001)$ of OSI decrease with improvement in CDVA that was similar to our study. McMillin et $\mathrm{al}^{40}$ retrospectively compared subjective grading to double-pass retinal imaging, measuring a mean decrease in OSI following YAG capsulotomy of $2.84 \pm 0.76(n=26, p=0.001)$, greater than the mean improvement in our study as they only had a few $(n=4)$ eyes with low $(1+)$ grading. In their series, patients with good initial BCVA (0.10 logMAR or better, $\mathrm{n}=10$ ) but with symptoms of glare and halos also showed significant improvement in OSI $(-0.76 \pm 0.16, \mathrm{p}=0.001)$ after capsulotomy, whereas the change in BCVA $(-0.02 \pm$ 0.02, $\mathrm{p}=0.34$ ) was not significant.

Using the HD Analyzer, we were able to measure optical quality before and after intervention via YAG capsulotomy in patients with PCO. Changes in HD Analyzer metrics were compared to changes in high and low contrast uncorrected and best-corrected visual acuity to permit for correlation of HD Analyzer metrics to visual acuity improvement. Other studies have employed the HD Analyzer to provide insight into cataract grading and severity, ${ }^{24-27}$ retinal image quality with cataract surgery, ${ }^{16}$ keratitis, ${ }^{22}$ keratoconus, ${ }^{23}$ and dry eyes. ${ }^{19-21}$ However, these studies have not described the correlation between the HD Analyzer parameters and both high and low contrast visual acuity.

We demonstrate here a moderate correlation of OSI to degradation of visual acuity due to PCO. Our data suggest that a scale using OSI can be implemented to predict the effect of PCO on visual function and, correspondingly, the potential utility of YAG capsulotomy, as a linear regression calculates no improvement in HC-BCVA if the preYAG OSI is less than or equal to 1.08 and at least one line improvement if the pre-YAG OSI is greater than or equal to 2.93 , with less than a line improvement expected if the pre-YAG OSI is between these two values. A similar linear regression calculates no significant clinical improvement 
in LC-BCVA if the pre-YAG OSI is less than or equal to 0.98 , at least one line improvement if the pre-YAG OSI is greater than or equal to 2.02, and at least two lines of improvement if the pre-YAG OSI is greater than or equal to 3.05. Hence, we propose the following preoperative HD Analyzer parameters may be used to grade PCO in patients with monofocal IOLs:

$-\mathrm{OSI} \leq 2.01$ mild PCO

-OSI 2.02 to 3.04 moderate PCO

-OSI $\geq 3.05$ severe PCO

The above grading system likely provides better clinical utility in deciding whether to perform a YAG capsulotomy, as low contrast vision more closely represents the visual demand many patients experience on a daily basis.

YAG capsulotomy is not without risks. Although none of our patients experienced worsening HC-BCVA, two of the patients had worsening LC-BCVA, one of which had a pre-OSI of less than 1.0. One patient had the CrystalensAT while the other had a standard monofocal IOL. Neither had signs of IOL decentration/dislocation, capsulotomy phimosis, retinal tears/detachments, or complaints of floaters. Thus, having objective data that provides prediction of visual acuity improvement, as we have in this paper, helps weigh the risks and benefits of performing a YAG capsulotomy. We also show that subjective PCO grading is not a good predictor of visual impact, as it does not take into account that forward scatter of light toward the fovea.

The pre-YAG OSI was predictive of approximately $60 \%$ of the change in HC-BCVA. Other factors that may influence the change can include IOL material and design, IOL thickness, IOL tilt, tear film changes, presence of nanoglistenings, ${ }^{41}$ or variation in internal scatter from ocular media opacities. Also, the HD Analyzer uses monochromatic infrared light for measure of scatter, which may not adequately represent normal polychromatic light in regard to the relative amount of scatter. To that point, some investigators using in vitro recordings have described angular and wavelength dependence of PCO-related straylight, which varied upon the nature and spatial distribution of the PCO. ${ }^{42}$ The HD Analyzer system cannot identify the anatomical source of decreased visual quality. ${ }^{15}$ While intra- and inter-session repeatability has been established, ${ }^{29}$ dynamic factors such as tear film quality may cause variation in measurements. ${ }^{21}$ Thus, it is imperative that these variables be minimized, such as by application of an artificial tear immediately prior to measurement as we utilized in this study. While the HD Analyzer system allows for control of the exit aperture, intra- and inter-measurement pupil size variation must be taken into account if miosis occurs and so measurements taken with a natural pupil of under 4 $\mathrm{mm}$ would not be comparable. In addition, while the HD analyzer presents metrics of the patient's retinal point spread function, it does not take into account individual variability in neural processing of the percept which also plays an important role in the formulation of high and low contrast visual acuity.

One weakness of the study is the lower limit of the ETDRS chart used for vision testing was 20/16 (-0.1 log MAR). Theoretically, a larger visual acuity improvement may have been demonstrated if patients were tested with charts that displayed smaller optotypes. Furthermore, only a small number of eyes were included in this study and we did not assess the morphology of the PCO. Future studies with larger study populations may be explored with the HD Analyzer system. In addition, previous studies of objective scatter in pseudophakic patients without PCO indicate that apodized and non-apodized multifocal IOLs have greater mean OSI values than monofocal IOLs ${ }^{43,44}$ and so specific predictive OSI cutoffs for Nd:YAG capsulotomy may need to be customized for specific IOL models, as well as the amount of angle kappa which appears to contribute to scatter metrics, independent of capsular clarity, in patients with multifocal or depth of focus IOLs. ${ }^{45}$

The strengths of this study include its prospective nature and the inclusion of patients with minimal capsular fibrosis who were being treated prophylactically to prevent Crystalens-related z-syndrome. This allowed us to establish lower thresholds for HD analyzer metrics that were predictive of high and low contrast visual gain, providing better guidance for these situations where the risk to benefit ratio may be otherwise less clear.

\section{Conclusions}

The HD Analyzer provides objective measurement of visual quality that can be used to quantify image degradation due to posterior capsular opacification and consequent improvement with surgical intervention. The ability to directly measure the forward scatter of light as it travels through the optical system using the HD Analyzer system provided higher correlation to post-capsulotomy changes in high and low contrast visual acuity rather than subjective slit-lamp assessment of posterior capsular fibrosis via backscattered light. Our data suggest specific preoperative OSI cutoffs that may predict the likelihood of 
improvement in high and low contrast vision following $\mathrm{Nd}$ :YAG laser capsulotomy in patients with monofocal and accommodating intraocular lenses.

\section{Data Sharing Statement}

The data sets generated and/or analyzed during the current study are not publicly available owing to the need to minimize risk to the privacy and confidentiality of research participants and ensure compliance with legal requirements for privacy and data protection, but are available from the corresponding author on reasonable request.

\section{Ethics and Consent Statement}

This trial was compliant with the principles of the Declaration of Helsinki and the International Conference on Harmonization guidelines for Good Clinical Practices. Written consent was obtained from each patient prior to any study-related procedures. The study protocol and its amendments, and the informed consent form, were reviewed and approved by the Southwest Independent Investigational Review Board (Houston, Texas).

\section{Disclosure}

The abstract and preliminary findings of this paper were presented at the 2011 American Academy of Ophthalmology Annual Meeting in Orlando, Florida as a poster presentation of interim findings.

Dr Qazi is a consultant to CorneaGen. Dr Pepose is a consultant to AcuFocus, Allergan, Bausch + Lomb, Johnson \& Johnson Vision, Kala Pharmaceuticals, Keeler, MG Therapeutics, Mimetogen Pharmaceuticals, Novartis, Noveome Biotherapeutics, Ocunexus Therapeutics, Okogen, Shire, a Takeda company, Stuart Pharmaceuticals, Sun Pharma, and TearLab. There were no grants pertinent to this study. Dr. Pepose received personal fees from Acufocus, Allergan, Bausch + Lomb, Johnson \& Johnson Vision, Kala Pharmaceuticals, Keeler, MG Therapeutics, Mimetogen Pharmaceuticals, Novartis, Noveome Biotherapeutics, Ocunexus Therapeutics, Okogen, Shire, a Takeda company, Stuart Pharmaceuticals, Sun Pharma, and TearLab, outside the submitted work. None of the other authors have any financial disclosures.

\section{References}

1. Congdon N, Vingerling JR, Klein BE, et al. Prevalence of cataract and pseudophakia/aphakia among adults in the United States. Arch Ophthalmol. 2004;122:487-494.
2. Wormstone IM, Eldred JA. Experimental models for posterior capsule opacification research. Exp Eye Res. 2016;142:2-12. doi:10. 1016/j.exer.2015.04.021

3. Billotte C, Berdeaux G. Adverse clinical consequences of neodymium: YAG laser treatment of posterior capsule opacification. $J$ Cataract Refract Surg. 2004;30:2064-2071. doi:10.1016/j.jcrs.2004.05.003

4. Wesolosky JD, Tennant M, Rudnisky CJ. Rate of retinal tear and detachment after neodymium: YAG capsulotomy. J Cataract Refract Surg. 2017;43:923-928. doi:10.1016/j.jcrs.2017.03.046

5. Camparini M, Macaluso C, Reggiani L, Maraini G. Retroillumination versus reflected-light images in the photographic assessment of posterior capsule opacification. Invest Ophthalmol Vis Sci. 2000;41: 3074-3079.

6. Hirnschall N, Crnej A, Gangwani V, Findl O. Comparison of method to quantify posterior capsule opacification using forward or backward light scattering. J Cataract Refract Surg. 2014;40:728-735. doi:10.10 16/j.jcrs.2013.10.035

7. Findl O, Beuhl W, Menapace R, et al. Comparison of 4 methods for quantifying posterior capsular opacification. J Cataract Refract Surg. 2003;29:106-111. doi:10.1016/S0886-3350(02)01509-2

8. Hirnschall N, Neumayer T, Buehl W, Findl O. Reproducibility of analysis software for qualitative observation of Elschnig pearls. Ophthalmic Surg Lasers Imaging. 2010;41:507-511. doi:10.3928/ 15428877-20100726-02

9. Bender L, Spalton DJ, Uyanonvara B, et al. POCOman: new system for quantifying posterior capsule opacification. $J$ Cataract Refract Surg. 2004;30:2058-2063. doi:10.1016/j.jcrs.2004.05.010

10. Aslam TM, Dhillon B, Werghi N, et al. Systems of analysis of posterior capsular fibrosis. Br J Ophthalmol. 2002;86:1181-1186. doi:10.1136/bjo.86.10.1181

11. Jose RJM, Bender LE, Boyce JF, Heatley C. Correlation between the measurement of posterior capsular opacification severity and visual function. J Cataract Refract Surg. 2005;31:534-542. doi:10.1016/j. jcrs.2004.07.022

12. Hayashi K, Hayashi H, Nakao F, Hayashi F. Reproducibility of posterior capsular opacification measurement using Scheimpflug videophotography. J Cataract Refract Surg. 1998;24:1632-1635. doi:10.1016/S0886-3350(98)80355-6

13. Saika S, Miyamoto T, Ishida I, et al. Comparison of Scheimpflug images of posterior capsule opacification and histological findings in rabbits and humans. J Cataract Refract Surg. 2001;27:1088-1092. doi:10.1016/S0886-3350(00)00860-9

14. Tanaka Y, Kato S, Miyata K, et al. Limitation of Scheimpflug videophotography system in quantifying posterior capsule opacification after intraocular lens implantation. Am $J$ Ophthalmol. 2004; 137:732-735. doi:10.1016/j.ajo.2003.11.011

15. Güell JL, Pujol J, Arjona M, Díaz-Doutón F, Artal P. Optical quality analysis system: instrument for objective clinical evaluation of ocular optical quality. J Cataract Refract Surg. 2004;30:1598-1599. doi:10.1016/j.jcrs.2004.04.031

16. Alió JL, Schimchak P, Monte's-Mico' R, Galal A. Retinal image quality after microincision intraocular lens implantation. $J$ Cataract Refract Surg. 2005;31:1557-1560. doi:10.1016/j.jcrs.2005.01.026

17. Vilaseca M, Peris E, Pujol J, Borras R, Arjona M. Intra- and intersession repeatability of a double-pass instrument. Optom Vis Sci. 2010;87:675-681. doi:10.1097/OPX.0b013e3181ea1ad3

18. Martinez-Roda JA, Vilaseca M, Ondategui JC, et al. Optical quality and intraocular scattering in a healthy young population. Clin Exp Optom. 2011;94:223-229. doi:10.1111/j.1444-0938.2010.00535.x

19. Diaz-Valle D, Arriola-Villalobos P, Garcia-Vidal SE, et al. Effect of lubricating eyedrops on ocular light scattering as a measure of vision quality in patients with dry eye. $J$ Cataract Refract Surg. 2012;38:1192-1197. doi:10.1016/j.jcrs.2012.02.040

20. Tan C-H, Labbé A, Liang Q, et al. Dynamic change of optical quality in patients with dry eye disease. Invest Ophthalmol Vis Sci. 2015;56:2848-2854. doi:10.1167/iovs.14-15757 
21. Montes-Mico R, Alio JL, Charman WN. Postblink changes in the ocular modulation transfer function measured by a double-pass method. Invest Ophthalmol Vis Sci. 2005;46:4468-4473. doi:10.11 67/iovs.05-0609

22. Jimenez JR, Ortiz C, Perez-Ocon F, Jimenez R. Optical image quality and visual performance for patients with keratitis. Cornea 2009;28:783-788. doi:10.1097/ICO.0b013e318196703a

23. Ye C, Ng PKF, Jhanji V. Optical quality assessment in normal and forme fruste keratoconus eyes with a double-pass system: a comparison and variability study. $\mathrm{Br} J$ Ophthalmol. 2014;98:1478-1483. doi:10.1136/bjophthalmol-2013-304494

24. Lim SA, Hwang J, Hwang K-Y, Chung S-H. Objective assessment of nuclear cataract: comparison of double-pass and Scheimpflug systems. J Cataract Refract Surg. 2014;40(5):716-721. doi:10.1016/j. jcrs.2013.10.032

25. Ortiz D, Alio' JL, Ruiz-Colecha J, Oser U. Grading nuclear cataract opacity by densitometry and objective optical analysis. J Cataract Refract Surg. 2008;34:1345-1352. doi:10.1016/j.jcrs.2008.04.022

26. Pan A-P, Wang Q-M, Huang F, Huang J-H, Bao F-J, Yu A-Y. Correlation among lens opacities classification system III grading, visual function index-14, pentacam nucleus staging, and objective scatter index for cataract assessment. Am $J$ Ophthalmol. 2015;159:241-247. doi:10.1016/j.ajo.2014.10.025

27. Galliot F, Patel SR, Cochener B. Objective scatter index: working toward a new quantification of cataract? J Refract Surg. 2016;32:96102. doi:10.3928/1081597X-20151222-02

28. Xu -C-C, Xue T, Wang Q-M, Zhou Y-N, Huang J-H, Yu A-Y. Repeatability and reproducibility of a double-pass optical quality analysis device. PLoS One. 2015;10:e0117587. doi:10.1371/journal. pone. 0117587

29. Iijima A, Shimizu K, Kobashi H, Saito A, Kamiya K. Repeatability, reproducibility, and comparability of subjective and objective measurements of intraocular forward scattering in healthy subjects. Biomed Res Int. 2015;2015:925217. doi:10.1155/2015/925217

30. Saad A, Saab M, Gatinel D. Repeatability of measurements with a double-pass system. J Cataract Refract Surg. 2010;36:28-33. doi:10.1016/j.jcrs.2009.07.033

31. Congdon N, Fan H, Choi K, et al. Impact of posterior subcapsular opacification on vision and visual function among subjects undergoing cataract surgery in rural China: study of cataract outcomes and up-take of services (SCOUTS) in the Caring is Hip Project, Report 5. $\mathrm{Br} J$ Ophthalmol. 2008;92:598-603. doi:10.1136/ bjo. 2007.126714

32. Jardim D, Soloway B, Starr C. Asymmetric vault of an accommodating intraocular lens. J Cataract Refract Surg. 2006;32:347-350. doi:10.1016/j.jcrs.2005.08.021

33. Zhang H, Wang J. Visual quality assessment of posterior capsule opacification using optical quality analysis system (OQAS). J Ophthalmol. 2017;2017:9852195. doi:10.1155/2017/9852195
34. Yotsukura E, Torii H, Saiki M, Negishi K, Tsubota K. Effect of neodymium: YAG laser capsulotomy on visual function in patients with posterior capsule opacification and good visual acuity. $J$ Cataract Refract Surg. 2016;42:399-404. doi:10.1016/j.jcrs.2015. 11.042

35. van den Berg TJTP, Spekreijse H. Light scattering model for donor lenses as a function of depth. Vision Res. 1999;39:1437-1445. doi:10.1016/S0042-6989(98)00220-X

36. Findl O, Buehl W, Siegl H, Pinz A. Removal of reflections in the photographic assessment of PCO by fusion of digital retroillumination images. Invest Ophthalmol Vis Sci. 2003;44:275-280. doi:10.11 67/iovs.02-0619

37. van Bree MCJ, Zijlmans BLM, van den Berg TJTP. Effect of neodymium: YAG laser capsulotomy on retinal straylight values in patients with posterior capsular opacification. J Cataract Refract Surg. 2008;34:1681-1686. doi:10.1016/j.jcrs.2008.06.014

38. van Bree MCJ, van den Berg TJTP, Zijlmans BLM. Posterior capsule opacification severity, assessed with straylight measurement, as main indicator of early visual function deterioration. Ophthalmology. 2013;120:20-33. doi:10.1016/j.ophtha.2012.07.050

39. Lu C, Yu S, Song H, et al. Posterior capsular opacification comparison between morphology and objective visual function. $B M C$ Ophthalmol. 2019;19:40. doi:10.1186/s12886-019-1051-z

40. McMillin JC, Rocha KM, Barwell EL, Haddad JS, Waring GO. Objective evaluation of the vision quality in pseudophakic patients with posterior capsular opacification using double-pass retinal imaging. Arq Bras Oftalmol. 2019;82:189-194. doi:10.5935/00042749.20190039

41. Beheregaray S, Yamamoto T, Hiraoka T, Oshika T. Influence on visual function of forward light scattering associated with subsurface nanoglistenings in intraocular lenses. J Cataract Refract Surg. 2014;40:1147-1154. doi:10.1016/j.jcrs.2013.10.047

42. van Bree MCJ, van der Meulen IJE, Franssen L, Coppens JE, Zijlmans BLM, van den Berg TJTP. In-vitro recording of forward light-scatter by human lens capsules and different types of posterior capsule opacification. Exp Eye Res. 2012;96:138-146. doi:10.1016/j. exer.2011.12.008

43. Pepose JS, Qazi MA, Chu R, Stahl J. A prospective randomized clinical evaluation of 3 presbyopia-correcting intraocular lenses after cataract extraction. Am J Ophthalmol. 2014;158:436-446. doi:10.1016/j.ajo.2014.06.003

44. Castillo-Gómez A, Carmona-González D, Martínez-de-la-Casa JM, Palomino-Bautista C, García-Feijoo J. Evaluation of image quality after implantation of 2 diffractive multifocal intraocular lens models. $J$ Cataract Refract Surg. 2009;35:1244-1250. doi:10.1016/j.jcrs.20 09.02 .024

45. Fu Y, Kou J, Chen D, et al. Influence of angle kappa and angle alpha on visual quality after implantation of multifocal intraocular lenses. $J$ Cataract Refract Surg. 2019;45:1258-1264. doi:10.1016/j.jcrs.20 19.04.003
Clinical Ophthalmology

\section{Publish your work in this journal}

Clinical Ophthalmology is an international, peer-reviewed journal covering all subspecialties within ophthalmology. Key topics include: Optometry; Visual science; Pharmacology and drug therapy in eye diseases; Basic Sciences; Primary and Secondary eye care; Patient Safety and Quality of Care Improvements. This journal is indexed on PubMed
Central and CAS, and is the official journal of The Society of Clinical Ophthalmology (SCO). The manuscript management system is completely online and includes a very quick and fair peer-review system, which is all easy to use. Visit http://www.dovepress.com/ testimonials.php to read real quotes from published authors. 